\title{
Innovation and robust sensor product development with Design for Six Sigma
}

\author{
Dipl.-Ing. Winfried Korb (1), Dipl.-Phys. Helmut Kremer (2)
}

1) arteos GmbH, Seligenstädter Str.91, D-63500 Seligenstadt, Germany, w.korb@arteos.com

2) micro-part GmbH+Co.is.KG, Am Deutelsberg 6, D-65375 Oestrich-Winkel, h.kremer@micro-part.de

Design for Six Sigma (DFSS) is a product development process that uses metrics, data, statistics, and project management tools. The goal of Design for Six Sigma is that the new product should have robust performance. DFSS can be incorporated into an organization's existing product development process and should involve taking products all the way from concept to commercialization. The follow paper describes the sensor developing process of the company arteos GmbH / Germany. In the presentation we give more DFSS-details and show a case study of sensor developing with DFSS.

Key words: sensor development with Design for Six Sigma.

\section{Theory of Six Sigma}

The theory of Six Sigma based on measuring a process in terms of defects. A Six Sigma process means this processes are working nearly perfectly, delivering only 3.4 defects per million opportunities (DPMO) (see table 1).

A one sigma $(1 \sigma)$ process produces 691462.5 defects per million opportunities, which translates to a percentage of satisfactory outputs of only $30.854 \%$. That is a really poor performance.

A $3 \sigma$ process produces 66807.2 errors per million opportunities (delivering 93.319\% satisfactory outputs). That's much better, but we're always still wasting money and disappointing our customers.

Most German companies are operating at 3 to $4 \sigma$ quality levels. That means they could be losing up to $25 \%$ of their total revenue due to processes that deliver too many defects. These defects take up time and effort to repair as well as make customers unhappy.

\begin{tabular}{|c|c|c|}
\hline $\begin{array}{c}\text { Sigma } \\
\text { level }\end{array}$ & $\begin{array}{c}\text { defects per million } \\
\text { opportunities }\end{array}$ & $\begin{array}{c}\text { percent of output } \\
\text { defect free }\end{array}$ \\
\hline 6 & 3,4 & $99,99966 \%$ \\
\hline 5 & 230 & $99,97 \%$ \\
\hline 4 & 6.210 & $99,4 \%$ \\
\hline 3 & 66.800 & $93,3 \%$ \\
\hline 2 & 308.000 & $69,2 \%$ \\
\hline 1 & 690.000 & $31 \%$ \\
\hline
\end{tabular}

Tab. 1: Defects (DPMO) per Sigma level

The central idea of Six Sigma management is that if we can measure the defects in a process, we can systematically figure out ways to eliminate them, to approach a quality level of zero defects.

\section{Basics of Design for Six Sigma DFSS}

The basic concept of DFSS is to clearly understand the customer's requirements and translate them to the critical to quality characteristics of the product. Understanding the fundamentals of the product will provide unforeseen flexibility and speed in developing products. 
Design for Six Sigma is

- a process for re-designing existing products;

- a methodology for designing new products;

- a way to exceed customer expectations;

- a strategy for small developing costs;

- a proceed for best product quality.

DFSS process begins with market segmentation and business analysis, and ends with deploying the needs of customers into innovative, capable, robust product and service designs providing enhanced value propositions in the marketplace worldwide.

DFSS is a set of tools and techniques that will allow your designs to be right the first time, work better than existing and competing products, with manufacturing and supply chain support, and have greater market share. There are seven main important DFSS-tools:

(1) Customer tool: Voice of the customer (VOC) techniques. Process to understand the wishes of the customers.

(2) Management tool: Organization to assess their product portfolio and formulate their product strategy.

(3) Risk management tool: Techniques for risk management at the end of each phase. To enables judicial decisions, avoiding customer issues and unforeseeable costs.

(4) Statistic toll: Evaluation techniques, advanced statistical and experimental tools.

(5) QFD tool: Quality Function Deployment (QFD) is a quality planning method. QFD ensures that customer requirements flow into the product. The whole process from development over manufacturing up to sales and service is included.

(6) Communication tool: Standardized processes and templates facilitate communication between teams, the business and the customers.

(7) Project management tool: The structured product development process provides a clear understanding of deliverables on product requirements, timelines and budgets during the product development cycle.

The benefits of DFSS are (figure 1):

1.) faster developing time

2.) warranty cost reductions

3.) high performance / cost ratio

4.) quality designed in from the start
5.) revenue growth: customer delight, market share, volume, price

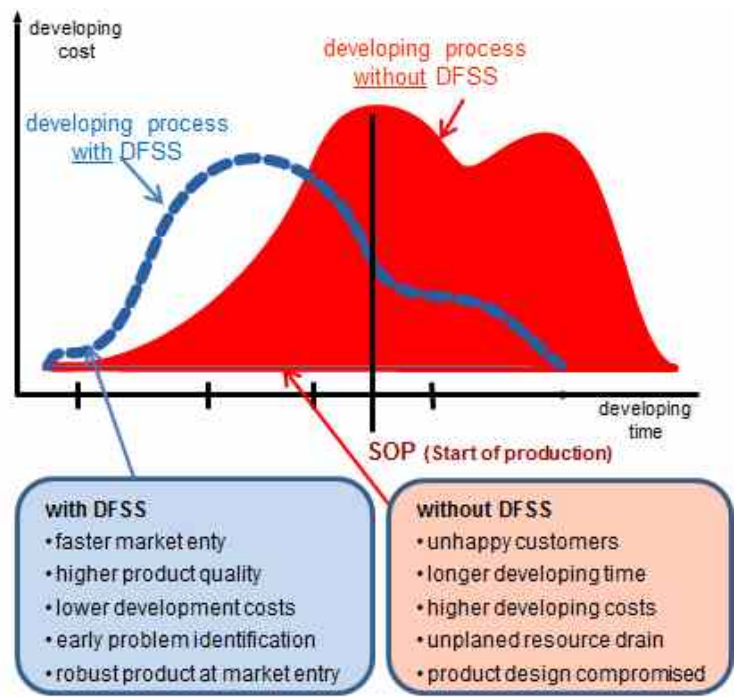

Fig. 1: Developing process with and without DFSS

The work with DFSS is a five step process, called DICOV:

1.) Define: Define the project and enable the team to succeed with the project by mapping all vital steps

2.) Identify: Hear the voice of the customer to select the best product concept.

3.) Characterize: Build a thorough knowledge base about the product and its processes

4.) Optimize: Achieve a balance of quality, cost, and time to market

5.) Validate: Demonstrate with data that the voice of the customer has been heard and that customer expectations have been satisfied

\section{3. arteos Sensor developing process}

In business unit "engineering" arteos develop their own or customer sensors. Here we will give a short overview of this arteos sensor developing process.

The developing is starting with three parallel developing processes. After these three basic processes the developing of production and assembly process will follow. Design for Six Sigma is the integrated and coordinated process of this sensor developing (figure 2). 


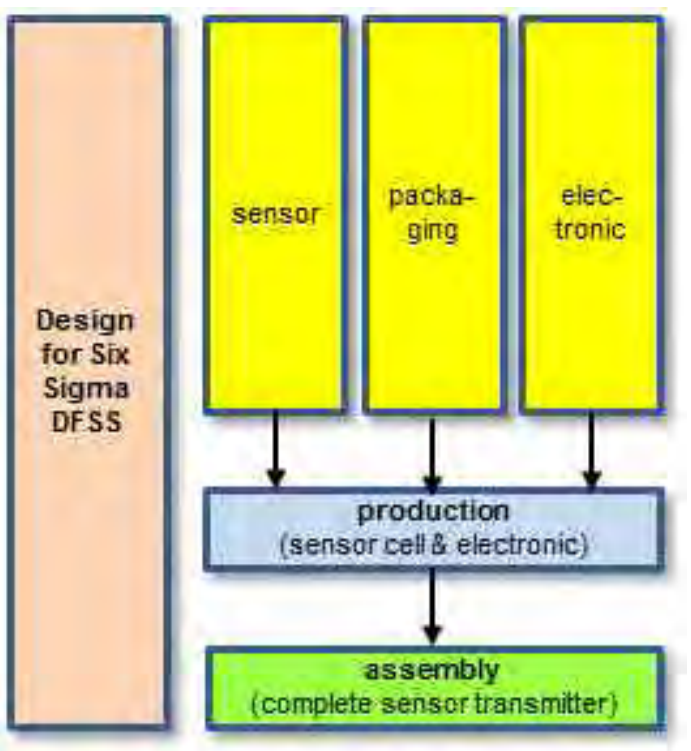

Fig. 3: Sensor developing process based on DFSS

The arteos sensor developing process based on DFSS and MiDes-Flow. MiDes-Flow (www.mides-kmu.de) is a structured developing process specialized for sensors and microsystems. MiDes-Flow is created by a BMBF R\&D-project (project-partner: micro-part, arteos, Uni Frankfurt, Uni Siegen, IMtech Rüsselsheim).

In the poster presentation we give more details about Design for Six Sigma in cooperation with MiDes-Flow and show a case study of sensor developing. 